\title{
Big Data Business Actual Analysis: Stock Price Prediction Based on Time Series Model
}

\author{
Aiwen Rui \\ Tianjin University of Finance and Economics, Tianjin 300222, China \\ Email: 3294679254@qq.com
}

\begin{abstract}
This paper selects the daily closing price data of the Shanghai Composite Index from January 1, 2016 to December 31, 2017, excluding holidays, and preprocesses the data. After taking the logarithm and converting it into the rate of return data, the first-order difference is performed to make it into a stable time series, and then the ARMA(p,q) model is constructed. Through parameter significance test, residual test and characteristic root test, according to the minimum principle of AIC, the optimal model is finally determined to be ARMA $(2,5)$ of sparse coefficient, and the expression of the model is obtained. The GARCH(1,1) model is established for the residual of ARMA(2,5), and the model expression is obtained. In order to directly predict the return rate of the Shanghai Composite Index, the ARIMA $(2,1,5)$ model of the sparse coefficient is constructed for the return rate of the Shanghai Composite Index, and the model expression is obtained. By predicting the Shanghai Composite Index return data on January 2, 2018, it is found that the prediction error of the model is small, and it can be used for subsequent predictions.
\end{abstract}

Keywords: ARMA(2,5) of sparse coefficient, $\operatorname{ARIMA}(2,1,5), \operatorname{GARCH}(1,1)$

\section{Construction of $\operatorname{ARMA}(p, q)$ model and residual $\operatorname{GARCH}(p, q)$ model}

\subsection{Data selection and preprocessing}

Due to the large volatility and differences of individual stocks in the stock market, using the Shanghai Stock Exchange Index as a reference standard to reflect the stock market situation can more systematically predict the stock market trend. Select the daily closing price data of the Shanghai Stock Exchange index from January 1, 2016 to December 31, 2017, excluding holidays, draw a relevant time series graph for the closing price data $\mathrm{y}$, and conduct an autocorrelation test, and find that the data is non-stationary.

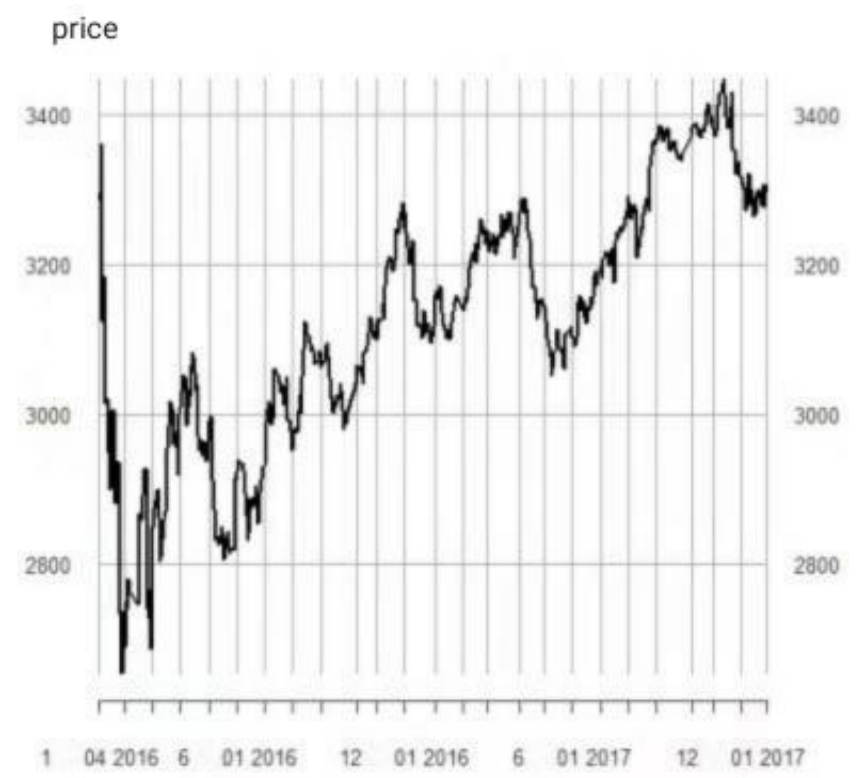

Figure 1. Time series chart of Shanghai stock index return rate from 2016 to 2017 

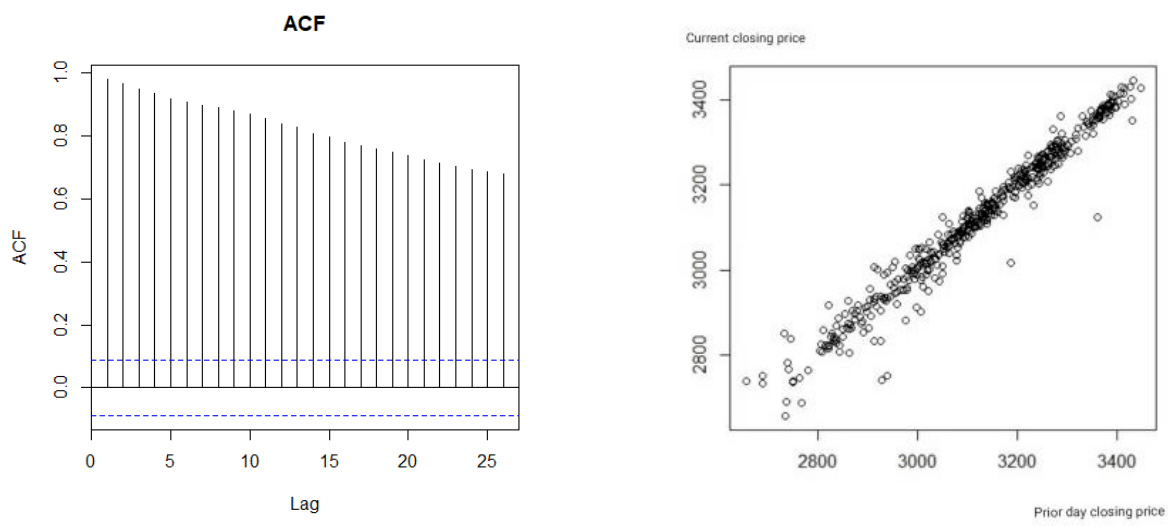

Figure 2. Autocorrelation graph of return rate and scatter plot of adjacent data

The general trend of the Shanghai Composite Index in the past two years has no obvious cycle and seasonality, and the fluctuation range is large. The ACF exhibits a tailing phenomenon, but the attenuation is slower and does not fall within the range. The adjacent data scatter chart shows the closing price of the previous day There is a clear positive correlation with the current closing price. Therefore, it can be judged that the sequence is non-stationary and does not satisfy randomness.

Next, the sequence is smoothed. Take the logarithm of the closing price sequence and transform it into a return rate sequence, and then perform a first-order difference to obtain the sequence $\nabla \ln y$, namely:

$$
\nabla \ln y=\ln y_{t}-\ln y_{t-1}, t=1,2,3, \cdots, n
$$

\subsection{Stationarity test}

It can be seen from the time series diagram that the time series after the first-order difference fluctuates at the zero mean value, and the ACF quickly decays to 0 , and basically all fall within the interval, so the series is stable.
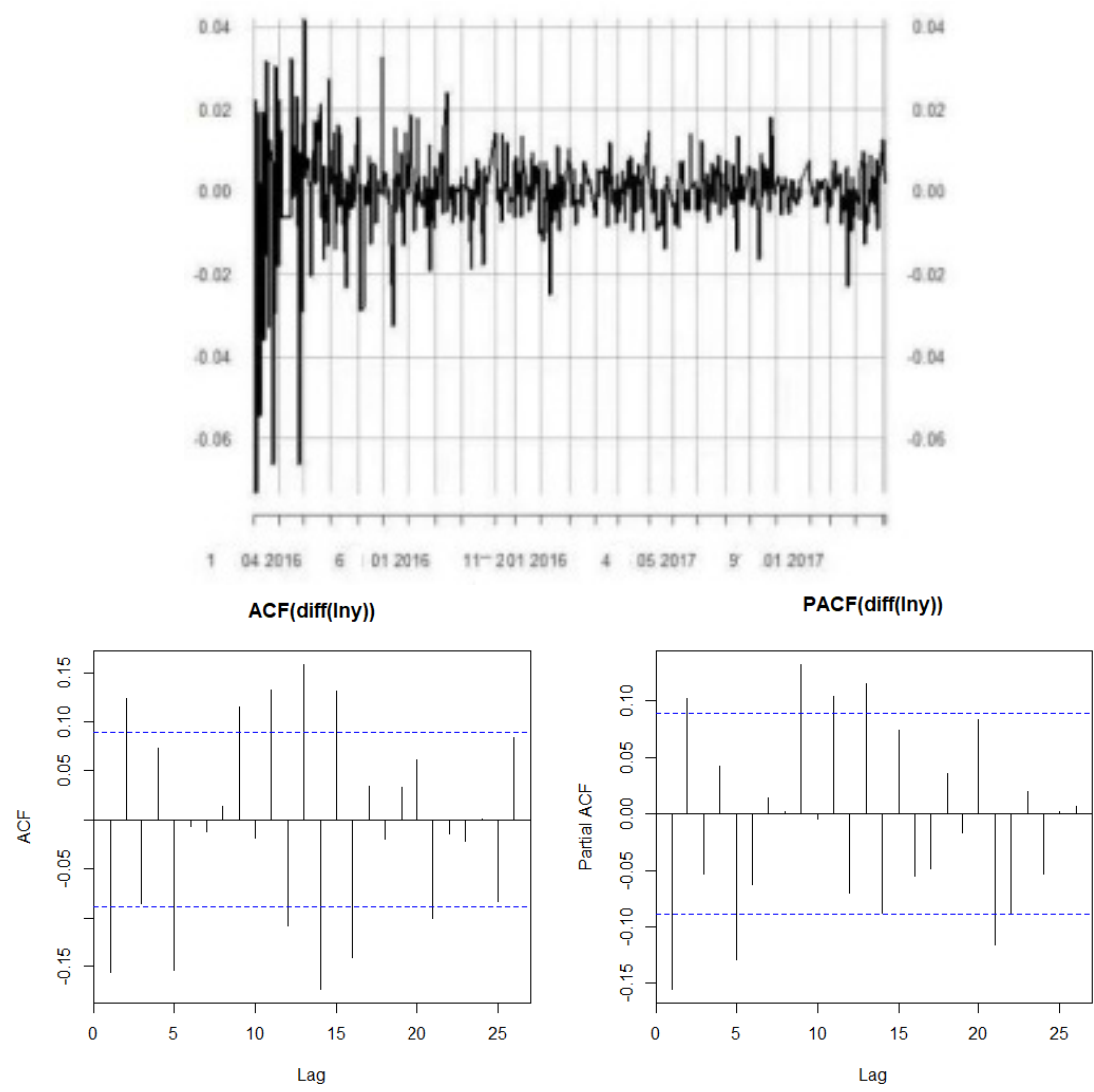

Figure 3. Time sequence diagram of return rate after first-order difference and ACF and PACF diagrams 


\subsection{White noise inspection}

Table 1. White noise test of yield series after first-order difference

\begin{tabular}{ccc}
\hline & Box- pierce test \\
\hline diff $(\ln x)-$ squared & 23.519 & 40.441 \\
$d f$ & 6 & 12 \\
$p$-value & 0.00064 & $6.07 \times e^{-5}$ \\
\hline
\end{tabular}

At the 5\% significance level, the null hypothesis is rejected, that is, the yield data after the first-order difference is not a white noise sequence.

\subsection{Construction of $\operatorname{ARMA}(\mathbf{p}, q)$ model}

Since the yield data after the first-order difference is stable and non-white noise, the ARMA(p,q) model is selected to fit the data.

\subsubsection{The order of the model}

After the above-mentioned smoothing treatment, the order is further determined by eacf.

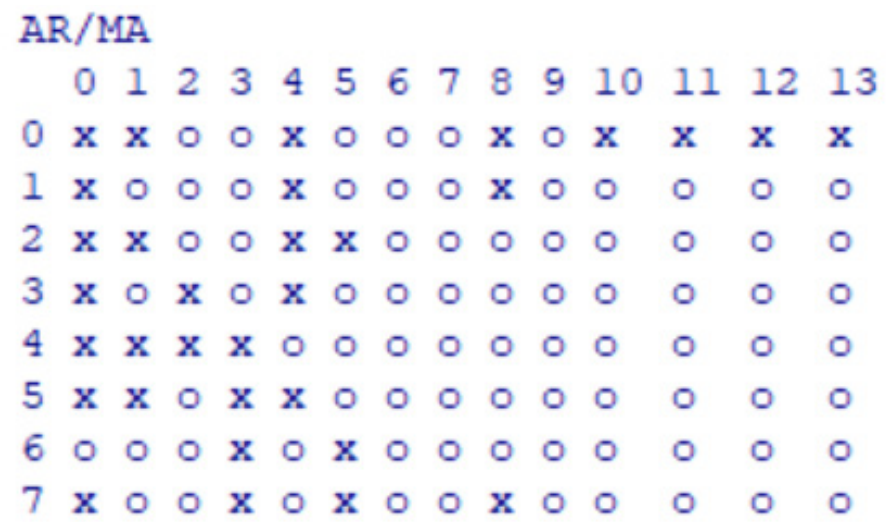

Figure 4. ARMA(p,q) model ordering diagram

It is preliminarily determined that the model is $\operatorname{ARMA}(3,5)$ or $\operatorname{ARMA}(1,5)$ model.

\subsubsection{Model parameter estimation and testing}

(1) Test and estimate the parameters of the model

Fit the data based on the $\operatorname{ARMA}(3,5)$ and $\operatorname{ARMA}(1,5)$ models, and adjust the parameters to make the model pass the significance test. The model $\operatorname{ARMA}(3,5)$ is reduced to the $\operatorname{ARMA}(2,5)$, the model $\operatorname{ARMA}(1,5)$ is reduced to $\mathrm{MA}(5)$.

Table 2. Parameter estimation results of $\operatorname{ARMA}(p, q)$ model

\begin{tabular}{|c|c|c|c|c|c|c|}
\hline Model & Parameter & Estimated value & Standard error & $\mathrm{T}$ test & P value & Significance \\
\hline \multirow{4}{*}{$\operatorname{ARMA}(2,5)$} & $\mathrm{AR}(2)$ & 0.48324 & 0.09590 & 5.039 & $4.68 \times e^{-7}$ & $* * *$ \\
\hline & $\operatorname{MA}(1)$ & -0.08508 & 0.04008 & -2.123 & 0.0338 & $*$ \\
\hline & $\operatorname{MA}(2)$ & -0.47600 & 0.10182 & -4.675 & $2.94 \times e^{-6}$ & $* * *$ \\
\hline & $\operatorname{MA}(5)$ & -0.11839 & 0.05146 & -2.301 & 0.0214 & $*$ \\
\hline \multirow{2}{*}{$M A(5)$} & $\operatorname{MA}(1)$ & -0.10452 & 0.04493 & -2.326 & 0.02000 & $*$ \\
\hline & $\operatorname{MA}(5)$ & -0.14120 & 0.04492 & -3.144 & 0.00167 & $* *$ \\
\hline
\end{tabular}

(2) Model checking and optimization

The white noise test on the residual sequence can verify the relevance of the model and the randomness of the variables, thereby verifying the rationality of the model. The residual sequence fluctuates around 0 , and the autocorrelation coefficient and partial autocorrelation coefficient coefficients quickly decay to 0 , and basically fall within the interval, so it can be judged that the residual is a stationary sequence. 


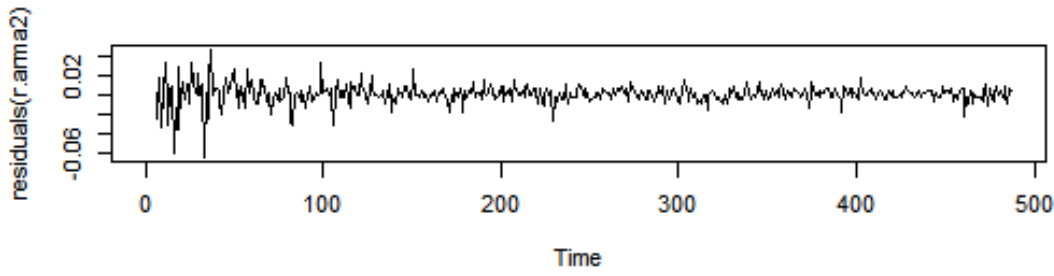

Series residuals(r.arma2)

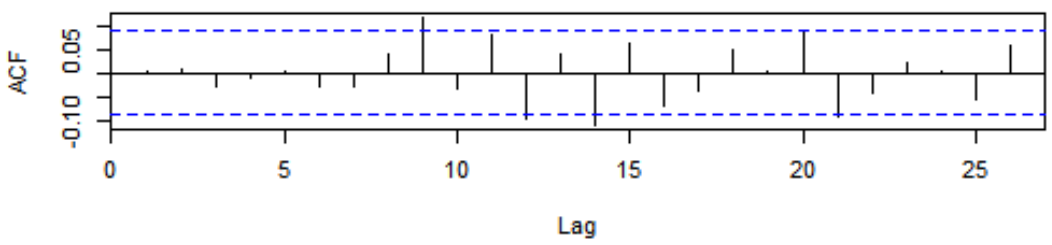

Series residuals(r.arma2)

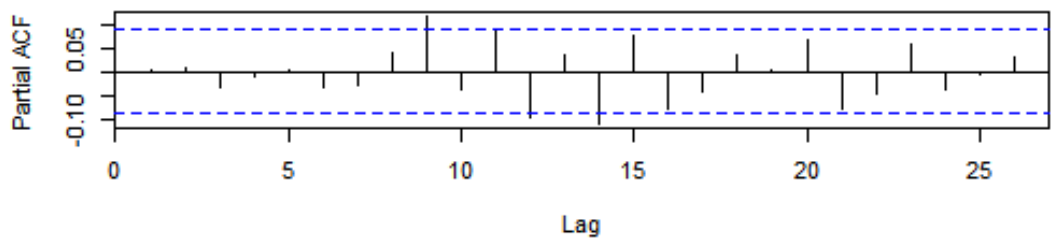

Figure 5. ARMA(2,5) residual sequence test chart

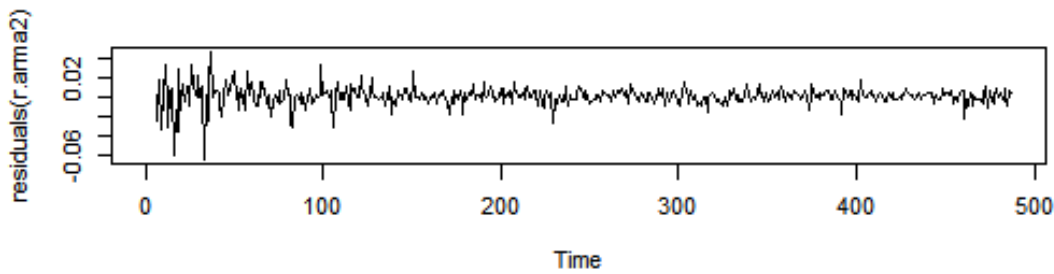

Series residuals(r.arma2)

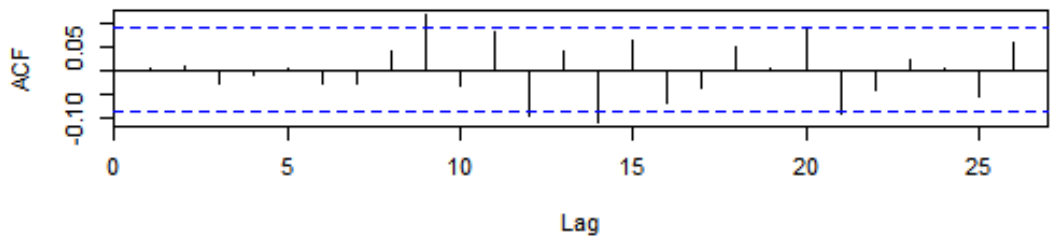

Series residuals(r.arma2)

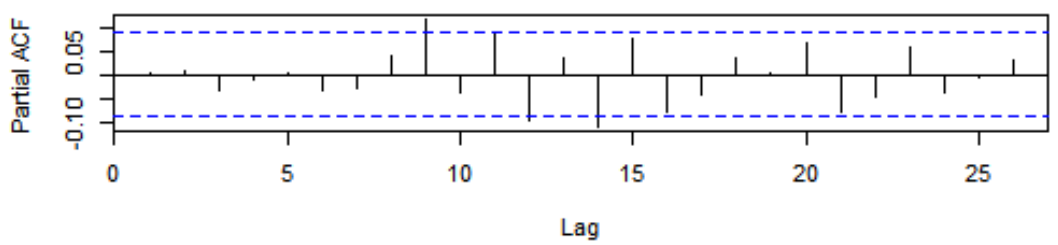

Figure 6. MA(5) residual sequence test chart 
Draw a scatter plot of $\operatorname{ARMA}(2,5)$ model and $\mathrm{MA}(5)$ residual adjacent data, and find that there is no correlation between the residual data and it is random.
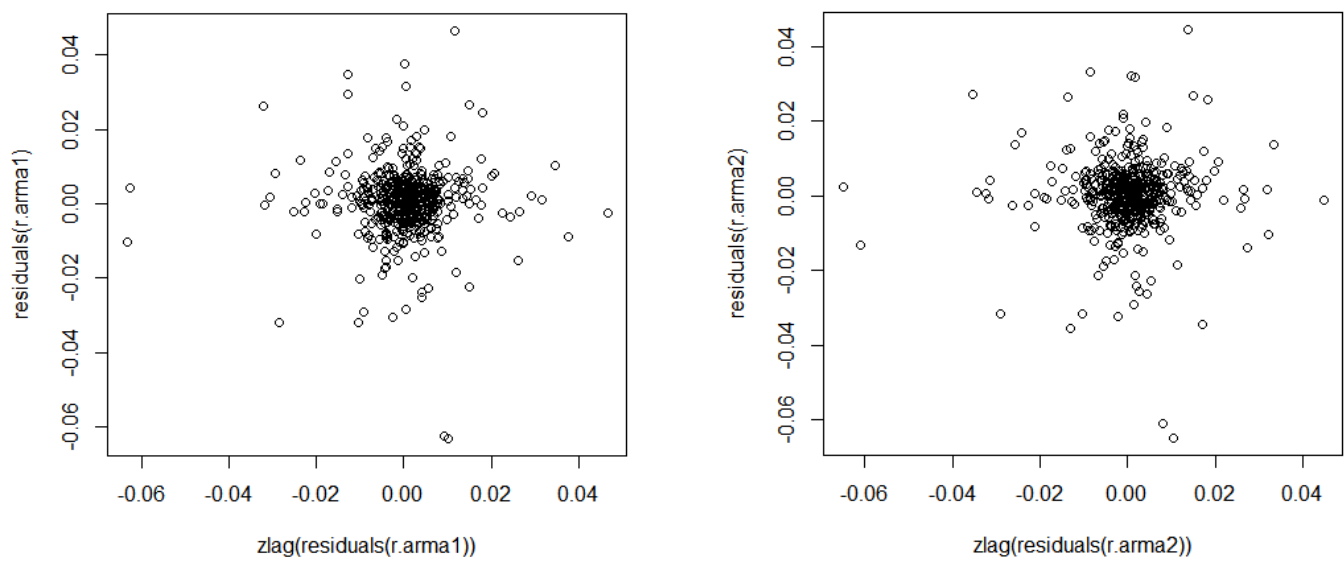

Figure 7. Scatter plot of adjacent data of residual sequence (left: $\operatorname{ARMA}(2,5)$; right: $\operatorname{MA}(5)$ )

Through Box test, the original hypothesis that the residual sequence is a white noise sequence is accepted at a significance level of $5 \%$, so the residual sequence is a white noise sequence.

Table 3. ARMA(2,5) residual sequence model parameter estimation results

\begin{tabular}{|c|c|c|c|}
\hline \multicolumn{4}{|c|}{ Box-pierce test } \\
\hline \multirow{3}{*}{$A R M A(2,5)$} & residuals(r.arma1) - squared & 1.5369 & 12.837 \\
\hline & $d f$ & 6 & 12 \\
\hline & $p$-value & 0.975 & 0.381 \\
\hline \multirow{3}{*}{$M A(5)$} & residuals(r.arma2) - squared & 0.99372 & 17.488 \\
\hline & $d f$ & 6 & 12 \\
\hline & $p$-value & 0.9858 & 0.1321 \\
\hline
\end{tabular}

In summary, the residuals of the $\operatorname{ARMA}(2,5)$ and $\mathrm{MA}(5)$ sequences are stationary white noise sequences, which proves that the conclusions of the model are valid, and the model already contains all the information of the data; in order to further test the rationality of the model, Discriminate the characteristic roots of the model, and find that the value of the characteristic roots of the two models is greater than 1, that is, all the characteristic roots are outside the unit circle. The model is determined to be the $\operatorname{ARMA}(2,5)$ of the sparse coefficient. The expression is:

$$
\left\{\begin{array}{c}
\widehat{d \ln y}=0.483239 X_{t-2}+\varepsilon_{t}-0.0850833 \varepsilon_{t-1}-0.4759967 \varepsilon_{t-2}-0.1183926 \varepsilon_{t-5} \\
\sigma_{\varepsilon}^{2}=9.009 \times e^{-5}
\end{array}\right.
$$

And the MA(5) of the sum sparse coefficient, the expression is:

$$
\left\{\begin{array}{c}
\widehat{d \ln y}=\varepsilon_{t}-0.1045183 \varepsilon_{t-1}-0.1412021 \varepsilon_{t-5} \\
\sigma_{\varepsilon}^{2}=9.243 \times e^{-5}
\end{array}\right.
$$

Choose the best model ARMA(2,5) through the AIC minimum principle.

\begin{tabular}{ccc}
\hline Model & ARMA(2,5) & MA(5) \\
\hline AIC & -3146.22 & -3146.22 \\
\hline
\end{tabular}

\subsection{ARMA(2,5) model prediction}

Next, use the model to simulate and predict the three working days from January 2 to 4, 2018. 
Table 4. ARMA(2,5) model prediction values

\begin{tabular}{ccc}
\hline Time & Observed value & $\operatorname{ARMA}(2,5)$ model prediction value \\
\hline 2018.01 .02 & 0.01236702 & 0.001051217 \\
2018.01 .03 & 0.06187650 & -0.00353813 \\
2018.01 .04 & 0.04915553 & 0.0008742533 \\
\hline
\end{tabular}

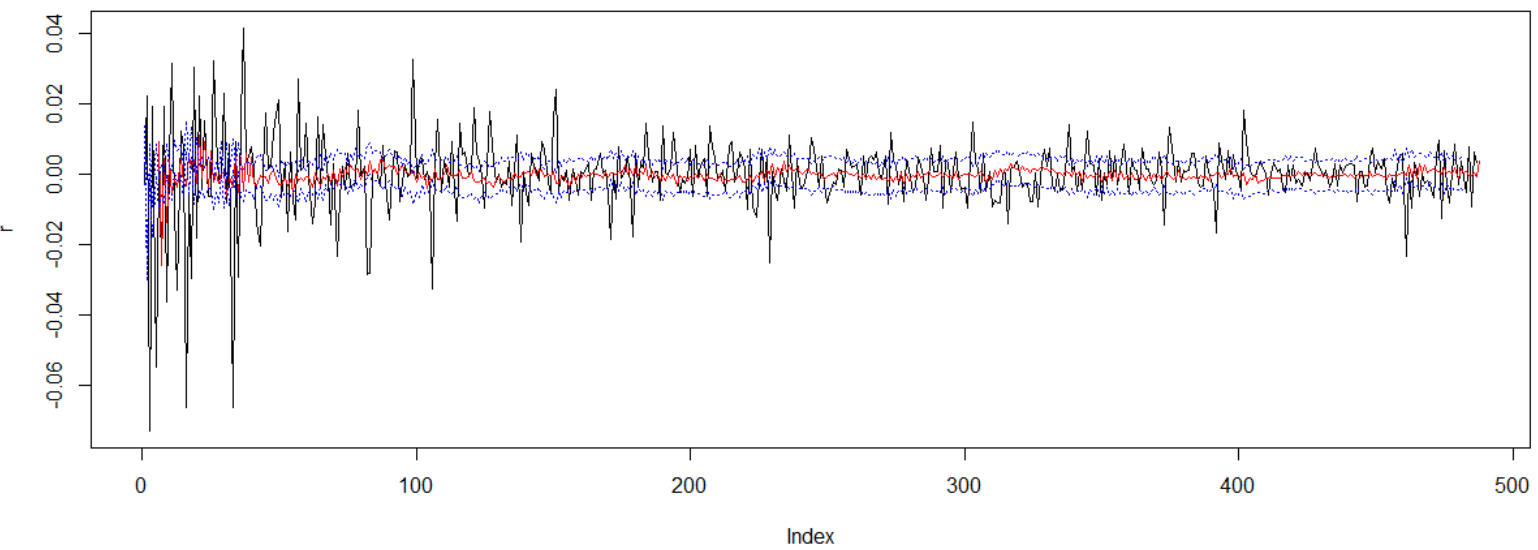

Figure 8. ARMA(2,5) model prediction graph

In Figure 8, the black curve represents the fitted sequence curve, the red curve represents the predicted value, and the blue dotted line represents the $95 \%$ confidence upper and lower limits of the fitted sequence, and the predicted value is basically within the confidence interval.

\subsection{Conditional heteroscedasticity modeling}

After obtaining the mean equation of the $\operatorname{ARMA}(2,5)$ model with sparse coefficients, the correlation test and conditional heteroscedasticity test are performed on the residual sequence of the model, and the GARCH(q,p) model is established for the sparse coefficient $\operatorname{ARMA}(2,5)$. The residual term of the model is fitted.

\subsubsection{Heteroskedasticity autocorrelation test}

Conditional heteroscedasticity is the ARCH effect. By drawing a time series diagram of the residuals, which is the sum of squares of the residuals, it is found that the series residuals have a fluctuating clustering effect. Afterwards, the residuals are tested and the test results are significant, most of which are less than 0.05 , indicating the model The residual sequence of has ARCH effect, you can try to build a GARCH model for the residuals.
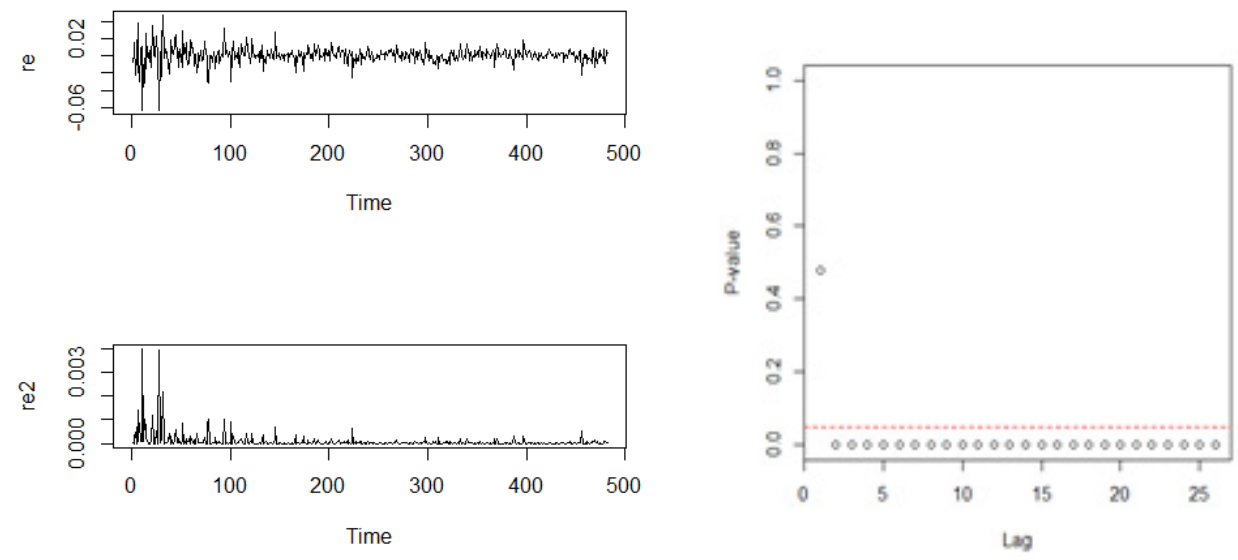

Figure 9. Residual sequence diagram and test diagram 


\subsubsection{GARCH model order determination and parameter estimation}

First, establish the ARMA(p,q) model to determine the order of the square of the residual.

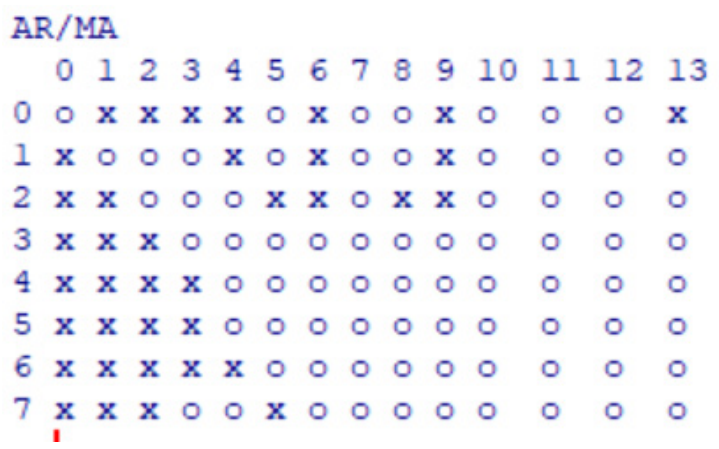

Figure 10. Model ordering diagram

Finally, the model is determined to be $\operatorname{ARMA}(1,1)$, and then the $\operatorname{GARCH}(1,1)$ model is fitted to the residual sequence. Adjust the parameters to make the model pass the significance test, and obtain the $\operatorname{GARCH}(1,1)$ model structure as:

$$
\varepsilon_{t \mid t-1}^{2}=6.751 \times e^{-7}+0.9391 \varepsilon_{t-1 \mid t-2}^{2}+4.814 \times e^{-2} \times \gamma_{t-1}^{2}
$$

Table 5. GARCH(1,1) model parameter estimation results

\begin{tabular}{cccccc}
\hline Parameter & Estimated value & Standard error & T test & P value & Significance \\
\hline $\mathrm{a}_{0}$ & $6.751 \times e^{-7}$ & $2.061 \times e^{-7}$ & 3.176 & 0.00105 & $* *$ \\
$\mathrm{a}_{1}$ & $4.814 \times e^{-2}$ & $7.798 \times e^{-3}$ & 6.174 & $6.68 \times e^{-10}$ & $* * *$ \\
$\mathrm{~b}_{1}$ & 0.9391 & $9.030 \times e^{-3}$ & 103.998 & $<2 \times e^{-3}$ & $* * *$ \\
\hline
\end{tabular}

\subsubsection{Normality test of residuals}

To test the residual of $\operatorname{GARCH}(1,1)$, the $\mathrm{p}$ value is less than 0.05 , and the null hypothesis of normality test cannot be accepted.

Table 6. Jarque Bera Checklist

\begin{tabular}{cc}
\hline \multicolumn{3}{c}{ Jarque Bera Test } \\
\hline$X-$ squared & 253.74 \\
$d f$ & 2 \\
$p$-value & $2.2 \times e^{-16}$
\end{tabular}

Through Box test, it is found that the p-values are all greater than 0.05 , which shows that the residuals of the GARCH(1,1) model are white noise sequences.

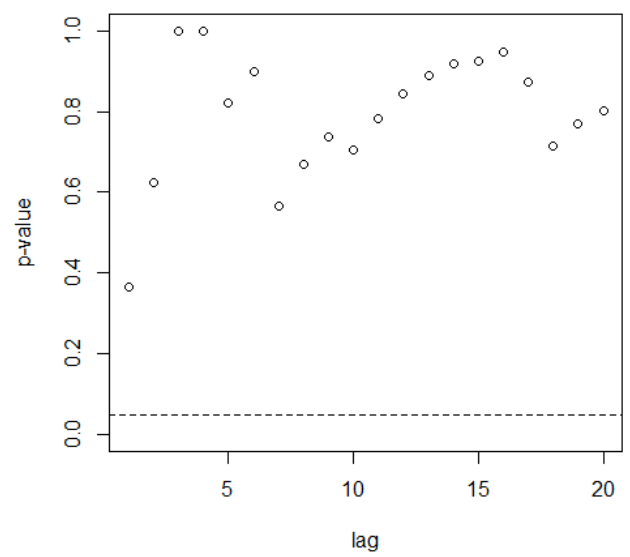

Figure 11. The residual Box test 
In summary, the ARMA(2,5)-GARCH(1,1) model is fitted to the return data after the first-order difference. The expression to get its mean and variance is:

$$
\begin{gathered}
\widehat{d \ln y}=0.483239 X_{t-2}+\varepsilon_{t}-0.0850833 \varepsilon_{t-1}-0.4759967 \varepsilon_{t-2}-0.1183926 \varepsilon_{t-5} \\
\varepsilon_{t \mid t-1}^{2}=6.751 \times e^{-7}+0.9391 \varepsilon_{t-1 \mid t-2}^{2}+4.814 \times e^{-2} \times \gamma_{t-1}^{2}
\end{gathered}
$$

\section{ARIMA (p, d, q) model construction and prediction}

Above we constructed the $\operatorname{ARMA}(2,5)$ model to predict the return sequence after the first-order difference, and constructed the GARCH(1,1) model for the residual items. In order to more intuitively predict the return rate of the Shanghai Composite Index in the next few days, we build an ARIMA(2,1,5) model for the non-stationary return rate series based on the above analysis.

\subsection{Unit root test}

In order to further test the stationarity of the return rate series, an ADF test is performed on the data. At a significance level of $1 \%$, the return rate series is not stable.

\begin{tabular}{cc}
\multicolumn{2}{c}{ Table 7. ADF inspection } \\
\hline \multicolumn{2}{c}{ Augmented Dickey-Fuller Test } \\
\hline Dickey-Fuller & -3.6401 \\
Lag order & 7 \\
$p$-value & 0.02889 \\
\hline
\end{tabular}

\subsection{ARIMA ( $p, d, q)$ model construction}

Based on the time series $\operatorname{ARMA}(2,5)$, by adjusting the parameters, the model passes the significance test, and the model is determined to be the $\operatorname{ARIMA}(2,1,5)$ of the sparse coefficient. The expression is:

$$
\left\{\begin{array}{c}
\nabla \overparen{\ln y}=0.3457 X_{t-2}+\varepsilon_{t}-0.1184 \varepsilon_{t-1}-0.2272 \varepsilon_{t-2}-0.1586 \varepsilon_{t-5} \\
\sigma_{\varepsilon}^{2}=0.0001061
\end{array}\right.
$$

Table 8. ARIMA(2,1,5) parameter estimation results

\begin{tabular}{ccc}
\hline Parameter & Estimated value & Standard Error \\
\hline$A R(2)$ & 0.3457 & 0.1843 \\
$M A(1)$ & -0.1184 & 0.0455 \\
$M A(2)$ & -0.2272 & 0.1748 \\
$M A(5)$ & -0.1586 & 0.0475 \\
$\widetilde{\sigma_{\varepsilon}^{2}}=0.0001061 ;$ Log likelihood value $=1537.26 ;$ AIC $=-3066.51$ & \\
\hline
\end{tabular}

\subsection{ARIMA(2,1,5) model prediction}

Use the ARIMA $(2,1,5)$ model to simulate and predict the return rate data for one working day on January 2, 2018, and draw the forecast map.

Table 9. ARIMA(2,1,5) predicted values

\begin{tabular}{cccc}
\hline Time & Observation value & ARIMA(2,1,5) model prediction value & Error ratio \\
\hline 2018.01 .02 & 8.10754 & 8.116216 & $0.107 \%$ \\
\hline
\end{tabular}




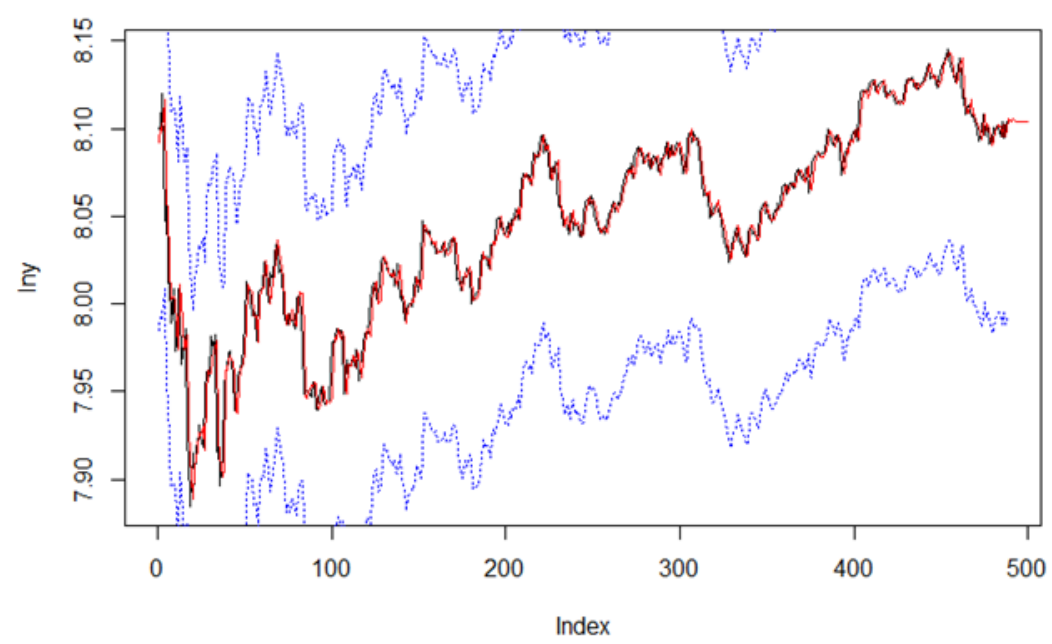

Figure 12. ARIMA(2,1,5) forecast chart

As can be seen from Figure 12, the black curve represents the fitted sequence curve, the red curve represents the predicted value, and the blue dashed line represents the $95 \%$ confidence upper and lower limits of the fitted sequence. The predicted value is basically within the range of the confidence interval, and the prediction is relatively accurate.

\section{Conclusion}

Fit the sparse coefficient $\operatorname{ARMA}(2,5)$ to the return data after the first-order difference, the expression of the model is: $\overparen{d \ln y}=0.483239 X_{t-2}+\varepsilon_{t}-0.0850833 \varepsilon_{t-1}-0.4759967 \varepsilon_{t-2}-0.1183926 \varepsilon_{t-5}$ And build the GARCH(1,1) model for the residuals of $\operatorname{ARMA}(2,5)$, and get the model expression as: $\varepsilon_{t \mid t-1}^{2}=6.751 \times e^{-7}+0.9391 \varepsilon_{t-1 \mid t-2}^{2}+4.814 \times e^{-2} \times \gamma_{t-1}^{2}$

In order to directly predict the rate of return of the Shanghai Composite Index, the ARIMA $(2,1,5)$ model of the sparse coefficient is constructed for the rate of return of the Shanghai Composite Index, and the model expression is obtained as:

$$
\nabla \overparen{\ln y}=0.3457 X_{t-2}+\varepsilon_{t}-0.1184 \varepsilon_{t-1}-0.2272 \varepsilon_{t-2}-0.1586 \varepsilon_{t-5}
$$

By predicting the Shanghai Composite Index return data on January 2, 2018, it is found that the prediction error of the model is small, and it can be used for subsequent predictions.

\section{References}

[1] Xu Jun. Empirical analysis of gold futures prices based on the ARMA model-523 sets of data from the New York Stock Exchange from 2006 to 2016. Industrial Economic Forum. 2017; 04(04): 16-22.

[2] Guo Xue, Wang Yanbo. Forecast of Shanghai stock index based on ARMA model. Times Economics and Trade. 2006; (S3): 58-59.

[3] Deng Jun, Yang Xuan, Wang Wei, Jiang Zhehui. Empirical research on stock price prediction using ARMA model. Enterprise Herald. 2010; (06): 266-267.

[4] Huang Lixia. Analysis and forecast of stock price based on ARIMA model — Taking Ping An of China as an example. Science and Technology Economic Market. 2020; (10): 62-63. 